\title{
Factors Influencing Oral Anticoagulant Prescribing Practices for Atrial Fibrillation
}

\author{
Lester Y. Leung, ${ }^{\mathrm{a}}$ Mark McAllister, ${ }^{\mathrm{b}}$ Magdy Selim, ${ }^{\mathrm{c}}$ Marc Fisher ${ }^{\mathrm{c}}$ \\ aDivision of Stroke and Cerebrovascular Diseases, Department of Neurology, Tufts Medical Center, Boston, MA, USA \\ ${ }^{b}$ Department of Neurosciences, Saint Elizabeth's Medical Center, Brighton, MA, USA \\ 'Division of Stroke and Cerebrovascular Diseases, Department of Neurology, Beth Israel Deaconess Medical Center, Boston, MA, USA
}

\section{Dear Sir:}

The factors influencing oral anticoagulant (OAC) prescription for atrial fibrillation (AF) are not well understood..$^{1-3}$ Novel oral anticoagulants (NOACS) have increased treatment options and complicated the decision making process for patients with AF. Prior studies identified factors predicting selection of NOACs over warfarin; however, prescriber knowledge and beliefs underlying these practices have not been evaluated. ${ }^{4-7}$ We sought to evaluate prescriber beliefs and trial interpretations influencing OAC prescription across specialties.

We conducted an anonymous, online survey with 9 multiple choice questions (Table 1). This study was approved by the Institutional Review Board (IRB) at Beth Israel Deaconess Medical Center. The first question asked respondents to identify their specialty and practice setting. The remaining questions explored beliefs and understanding of the medical literature. The survey was pilot tested on a focus group to assess understandability, length, and validity before broader distribution. The survey was administered using a secure, web-based application (www.SurveyMonkey.com). Participants were invited by e-mail and were contacted up to 3 times to complete the consent and survey.

We distributed the survey to 204 internists (hospital-based and community primary care physicians, hospitalists) and 42 cardiologists associated with Beth Israel Deaconess Medical Center and 185 Vascular Neurologists at U.S. academic medical centers.

Responses to survey questions were stratified by specialty. Comparisons between proportions of responses were analyzed with the Chi-square test with $\alpha=0.05$. To account for multiple comparisons, a Bonferroni corrected $P$-value of 0.0035 is pre- sented (significance denoted by ${ }^{*}$ in the Results section).

128 of 431 potential participants (30\%) completed the survey (58 of 204 Internists, 16 of 42 Cardiologists, 54 of 185 Vascular Neurologists). Among internists, there were 22 community primary care physicians, 29 hospital-based primary care physicians, and 7 hospitalists.

Interpretation of the term "stroke" varied, though not between specialties: a majority in each specialty (60\% internists, $69 \%$ cardiologists, $83 \%$ neurologists, $P=0.027$ ) selected a broad definition including ischemic stroke (IS) and several forms of hemorrhage. A minority (31\% internists, 31\% cardiologists, 17\% neurologists, $P=0.18$ ) selected a definition limited to IS. When prescribing anticoagulation, most (66\%) were primarily concerned with IS prevention efficacy (60\% internists, 69\% cardiologists, $70 \%$ neurologists, $P=0.52$ ) instead of safety, cost, or ease of reversal.

There were no major differences between specialties regarding concern about major bleeding risk with warfarin (13\% cardiologists, 36\% internists, 31\% neurologists) $(P=0.19)$. Regarding combining warfarin with aspirin, neurologists $(70 \%)$ were more likely $\left(P=0.00028^{*}\right)$ to consider warfarin sufficient for patients with AF and coronary artery disease without stents or to avoid combining medications as compared to internists (40\%) or cardiologists (25\%). Internists (45\%) and cardiologists (56\%) frequently prescribed this combination despite their concerns about bleeding risk; this was uncommon among neurologists (15\%) ( $\left.P=0.00043^{*}\right)$.

Regarding the comparative efficacy of NOACs and warfarin, all specialties were equally likely to be unaware that only dabigatran has demonstrated superiority compared to warfarin in IS prevention in clinical trials: some internists $(45 \%)$, cardiologists 
Table 1. Survey questions and responses

\begin{tabular}{|c|c|c|c|c|}
\hline & Internal Medicine & Cardiology & Vascular Neurology & $P$ \\
\hline \multicolumn{5}{|l|}{ 1. My clinical practice best described by the following: } \\
\hline a. Primary care at an academic center & 29 & 0 & 0 & \\
\hline b. Primary care in a community setting & 22 & 0 & 0 & \\
\hline c. Cardiology at an academic center & 0 & 16 & 0 & \\
\hline d. Cardiology in a community setting & 0 & 0 & 0 & \\
\hline e. Vascular neurology at an academic center & 0 & 0 & 54 & \\
\hline f. Vascular neurology in a community setting & 0 & 0 & 0 & \\
\hline g. Medicine hospitalist at an academic center & 7 & 0 & 0 & \\
\hline h. Medicine hospitalist in a community hospital & 0 & 0 & 0 & \\
\hline \multicolumn{5}{|c|}{ 2. To me, the work "stroke" encompasses which of the following diseases? } \\
\hline a. Ischemic brain infarction & $18 / 58$ & $5 / 16$ & $9 / 54$ & 0.18 \\
\hline b. Primary intracerebral hemorrhage $(\mathrm{ICH})$ & $5 / 58$ & 0 & 0 & $N P^{*}$ \\
\hline c. All types of intracranial hemorrhages & 0 & 0 & 0 & NP \\
\hline d. All of the above & $35 / 58$ & $11 / 16$ & $45 / 54$ & 0.027 \\
\hline
\end{tabular}

3. Do you believe that the ischemic stroke (IS) risk is the same for persistent and paroxysmal atrial fibrillation (AF)?

$\begin{array}{lrrr}\text { a. Yes } & 39 / 58 & 15 / 16 & 44 / 45 \\ \text { b. No } & 19 / 58 & 1 / 16 & 10 / 54 \quad \text { NP }\end{array}$

4. When I prescribe an anticoagulant to patients with $A F$, my primary consideration is:

\begin{tabular}{|c|c|c|c|c|}
\hline a. Efficacy of IS prevention & $35 / 58$ & $11 / 16$ & $38 / 54$ & 0.52 \\
\hline b. Safety profile of the anticoagulant (bleeding) & $18 / 58$ & $5 / 16$ & $9 / 54$ & NP \\
\hline c. Ease of use by patients & $4 / 58$ & 0 & $6 / 54$ & NP \\
\hline d. Cost & $1 / 58$ & 0 & $1 / 54$ & NP \\
\hline e. Ease of reversal of anticoagulation & 0 & 0 & 0 & NP \\
\hline \multicolumn{5}{|l|}{ 5. I am concerned with which one of the following when I use warfarin? } \\
\hline a. Risk of major bleeding & $21 / 58$ & $2 / 16$ & $17 / 54$ & 0.19 \\
\hline b. The need for frequent blood tests & 13/58 & $2 / 16$ & $3 / 54$ & NP \\
\hline c. Dietary restrictions & $1 / 58$ & 0 & $3 / 54$ & NP \\
\hline d. Difficulty effectively reversing the $A C$ effect in a timely manner & 0 & 0 & $1 / 54$ & NP \\
\hline e. Fluctuations of the INR & $23 / 58$ & $12 / 16$ & $30 / 54$ & 0.028 \\
\hline
\end{tabular}

6. Concerning the novel oral anticoagulants (NOACs) currently approved by the FDA, which of the following is INCORRECT?

\begin{tabular}{|c|c|c|c|c|}
\hline $\begin{array}{l}\text { a. In clinical trials, only dabigatran significantly reduced the risk of IS as } \\
\text { compared with warfarin. }\end{array}$ & $26 / 58$ & $3 / 16$ & $18 / 54$ & 0.13 \\
\hline $\begin{array}{l}\text { b. Dabigatran, rivaroxaban, and apixaban have reduced risk of ICH com- } \\
\text { pared to warfarin. }\end{array}$ & $10 / 58$ & $3 / 16$ & $4 / 54$ & NP \\
\hline $\begin{array}{l}\text { c. When combined with aspirin, dabigatran, rivaroxaban, and apixaban had } \\
\text { a similar increase in bleeding risk compared to the combination of aspirin } \\
\text { and warfarin. }\end{array}$ & 10/58 & $4 / 16$ & $5 / 45$ & NP \\
\hline $\begin{array}{l}\text { d. Dabigatran, rivaroxaban, and apixaban significantly reduce the risk of IS } \\
\text { compared to warfarin. }\end{array}$ & $12 / 58$ & $6 / 16$ & $27 / 54$ & 0.005 \\
\hline \multicolumn{5}{|c|}{ 7. When choosing a NOAC and not warfarin for stroke prevention in $A F$, my primary consideration is: } \\
\hline a. Better efficacy for preventing IS & $7 / 58$ & $2 / 16$ & $9 / 54$ & NP \\
\hline b. Reduced risk of intracranial hemorrhage & $6 / 58$ & $3 / 16$ & $27 / 54$ & $0.000013^{+}$ \\
\hline c. Lack of dietary restrictions & $1 / 58$ & $1 / 16$ & $2 / 54$ & NP \\
\hline d. No need for INR monitoring & $44 / 58$ & $10 / 16$ & $16 / 54$ & $<0.0001^{+}$ \\
\hline
\end{tabular}


Table 1. Continued

\begin{tabular}{|c|c|c|c|c|}
\hline & Internal Medicine & Cardiology & Vascular Neurology & $P$ \\
\hline \multicolumn{5}{|c|}{ 8. Which of the following is the most important impediment when you consider prescribing a NOAC? } \\
\hline a. Lack of a proven antidote to reverse $\mathrm{AC}$ effect & $21 / 58$ & $1 / 16$ & $21 / 54$ & 0.045 \\
\hline $\begin{array}{l}\text { b. Lack of readily available, rapid point-of-care blood test to assess degree } \\
\text { of AC effect afforded by the NOACs }\end{array}$ & $4 / 58$ & $1 / 16$ & $6 / 54$ & NP \\
\hline c. Cost to the patients, especially those on Medicare & $18 / 58$ & $13 / 16$ & $20 / 54$ & $0.0012^{+}$ \\
\hline $\begin{array}{l}\text { d. Lack of data in other conditions besides AF which I typically use warfarin } \\
\text { for stroke prevention }\end{array}$ & $5 / 58$ & 0 & $5 / 54$ & NP \\
\hline e. Concerns about increased bleeding in the elderly & $10 / 58$ & $1 / 16$ & $2 / 54$ & NP \\
\hline \multicolumn{5}{|l|}{ 9. My approach to combining warfarin with antiplatelet therapy is best exemplified by: } \\
\hline $\begin{array}{l}\text { a. I frequently use the combination in patients with } \mathrm{AF} \text { and coronary artery } \\
\text { disease without stents. }\end{array}$ & $10 / 58$ & $3 / 16$ & $8 / 54$ & NP \\
\hline $\begin{array}{l}\text { b. I believe warfarin alone is sufficient in patients with AF and coronary ar- } \\
\text { tery disease without stents. }\end{array}$ & $7 / 58$ & $2 / 16$ & $15 / 54$ & NP \\
\hline $\begin{array}{l}\text { c. I have major concerns about bleeding risk with this combination but still } \\
\text { prescribe them frequently. }\end{array}$ & $26 / 58$ & $9 / 16$ & $8 / 54$ & $0.00043^{+}$ \\
\hline $\begin{array}{l}\text { d. I rarely prescribe the combination in patients with } \mathrm{AF} \text { and coronary artery } \\
\text { disease without stents. }\end{array}$ & $15 / 58$ & $2 / 16$ & $23 / 54$ & NP \\
\hline Composite b+d ("avoidance") & $22 / 58$ & $4 / 16$ & $38 / 54$ & $0.00028^{+}$ \\
\hline Composite a+c ("frequent use") & $36 / 58$ & $12 / 16$ & $16 / 54$ & NP \\
\hline
\end{tabular}

$N P$, not performed; ICH, intracerebral hemorrhage; $A C$, anticoagulant; INR, international normalized ratio; FDA, Food and Drug Administration. *Statistical test not performed; 'Statistically significant with Bonferroni threshold of $P<0.0035$.

(19\%), and neurologists (33\%) thought this statement was false $(P=0.13)$. Neurologists $(50 \%)$ more often correctly identified that rivaroxaban and apixaban have not demonstrated superiority compared to warfarin in IS prevention compared to internists $(21 \%)$ and cardiologists $(38 \%)(P=0.005)$.

When selecting NOACs, internists (76\%) and cardiologists $(63 \%)$ were more likely than neurologists (30\%) to cite the lack of international normalized ratio monitoring as the primary benefit $\left(P<0.00001^{*}\right)$. Neurologists were more likely to cite a reduced risk of intracerebral hemorrhage $(\mathrm{ICH})$ as the primary advantage of NOACs (50\%) compared to internists (10\%) and cardiologists (19\%) $\left(P=0.000013^{*}\right)$.

Reasons for hesitancy in selecting NOACs varied across specialties. Cardiologists (6\%) were less concerned about the lack of an antidote compared to internists (36\%) and neurologists (39\%) $(P=0.045)$. Cardiologists were more concerned about cost $(81 \%)$ compared to internists (45\%) and neurologists (59\%) $\left(P=0.0012^{*}\right)$.

Attitudes toward OAC selection are heterogeneous across specialties. These specialties had different perceptions of the advantages and disadvantages of NOACs compared to warfarin, and they differed in their use of aspirin combined with warfarin. Overall, Neurologists were more concerned with hemorrhage risk as evidenced by their avoidance of the combination of aspirin and warfarin and their selection of NOACs for intracerebral hemorrhage risk reduction.

Surprisingly, there were no differences between specialties regarding the inclusiveness of the term "stroke," and many had difficulty in identifying differences in IS prevention efficacy. This variable interpretation of "stroke" is troublesome regarding outcome reporting in clinical trials. Clinicians and patients may misinterpret the universal reduction of intracerebral hemorrhage and its effect on the composite outcome of all strokes as superiority in IS reduction for all NOACs. This misconception may have important effects on risk counseling. In the original trials, only dabigatran was superior to warfarin in IS reduction, but the risk of gastrointestinal hemorrhage was greater. Physicians and patients at low risk for gastrointestinal hemorrhage may select dabigatran based on IS prevention efficacy, whereas they may select another NOAC in situations where hemorrhage risk aversion is a higher priority.

Our study has several strengths. Few published studies have evaluated prescription practices across specialties: this study encompasses the disciplines most likely to prescribe OACs for AF. The sample of neurologists includes clinicians throughout the U.S.; the Internists represented a variety of practice settings. This study also has limitations. The survey response rate was modest. However, the sample was sufficient to detect differences between specialties. Response rates in comparable electronic survey studies are highly variable. ${ }^{8-10}$ Finally, the survey assesses at- 
titudes and approaches but does not directly measure prescriptions; this may reflect opinions but not actual clinical practices.

In conclusion, this study provides an exploratory analysis of differences in beliefs influencing prescription practices of OACs. Educational interventions may prevent misinterpretation of study results, especially regarding composite outcomes. Trial results should use precise outcome definitions to avoid misleading clinicians and patients. The field of anticoagulation is rapidly changing: more shifts in decision making may follow the development of NOAC reversal agents. Even with these medications, comparative effectiveness studies and shared decision making tools will be needed to guide physicians and their patients.

\section{References}

1. Hsu JC, Chan PS, Tang F, Maddox TM, Marcus GM. Differences in anticoagulant therapy prescription in patients with paroxysmal versus persistent atrial fibrillation. Am J Med 2015;128:654.e1-654.e10.

2. Cowan $C$, Healicon R, Robson I, Long WR, Barrett J, Fay M, et al. The use of anticoagulants in the management of atrial fibrillation among general practices in England. Heart 2013;99:1166-1172.

3. Deplanque D, Leys D, Parnetti L, Schmidt R, Ferro J, de Reuck $J$, et al. Secondary prevention of stroke in patients with atrial fibrillation: factors influencing the prescription of oral anticoagulation at discharge. Cerebrovasc Dis 2006;21:372-379.

4. Xu Y, Holbrook AM, Simpson CS, Dowlatshahi D, Johnson AP. Prescribing patterns of novel oral anticoagulants following regulatory approval for atrial fibrillation in Ontario, Canada: a population-based descriptive analysis. CMAJ Open 2013;1:E115-E119.

5. Desai NR, Krumme AA, Schneeweiss S, Shrank WH, Brill G, Pezalla EJ, et al. Patterns of initiation of oral anticoagulants in patients with atrial fibrillation- quality and cost implications. Am J Med 2014;127:1075-1082.e1.

6. Luger $S$, Hohmann $C$, Kraft $P$, Halmer R, Gunreben I, Neumann-Haefelin $T$, et al. Prescription frequency and predictors for the use of novel direct oral anticoagulants for secondary stroke prevention in the first year after their marketing in Europe--a multicentric evaluation. Int J Stroke 2014;9:569575.

7. Albert NM. Use of novel oral anticoagulants for patients with atrial fibrillation: systematic review and clinical implications. Heart Lung 2014;43:48-59.

8. Cockroft KM, Chang KE, Lehman EB, Harbaugh RE. AVM management equipoise survey: physician opinions regarding the management of brain arteriovenous malformations. $J$ Neurointerv Surg 2014;6:748-753.

9. Provenzano DA, Deer T, Luginbuhl Phelps A, Drennen ZC, Thomson $S$, Hayek SM, et al. An international survey to understand infection control practice for spinal cord stimulation. Neuromodulation 2016;19:71-84.

10. Evans RW, Ghosh K. A survey of headache medicine specialists on career satisfaction and burnout. Headache 2015;55:14481457.

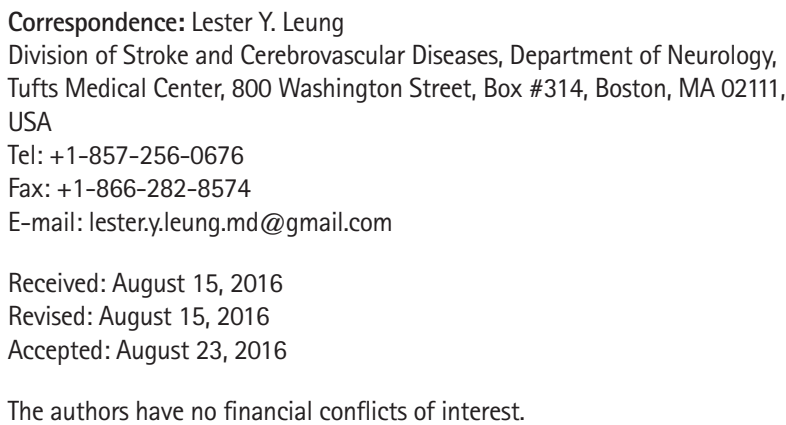

